\title{
F-PURITY AND RATIONAL SINGULARITY IN GRADED COMPLETE INTERSECTION RINGS
}

\author{
RICHARD FEDDER
}

\begin{abstract}
A simple criterion is given for determining "almost completely" whether the positively graded complete intersection ring $R=K\left[X_{1}, \ldots, X_{n+t}\right] /\left(G_{i}, \ldots, G_{t}\right)$, of dimension $n$, has an $F$-pure type singularity at $m=\left(X_{1}, \ldots, X_{n+t}\right)$. Specifically, if $\operatorname{deg}\left(X_{i}\right)=\alpha_{i}>0$ for $1 \leqslant i \leqslant n+t$ and $\operatorname{deg}\left(G_{i}\right)=d_{i}>0$ for $1 \leqslant i \leqslant t$, then there exists an integer $\delta$ determined by the singular locus of $R$ such that:

(1) $R$ has $F$-pure type if $\sum_{i=1}^{t} d_{i}-\sum_{i=1}^{n+t} \alpha_{i}<\delta$.

(2) $R$ does not have $F$-pure type if $\sum_{i=1}^{t} d_{i}-\sum_{i=1}^{n+t} \alpha_{i}>0$

The characterization given by this theorem is particularly effective if the singularity of $R$ at $m$ is isolated. In that case, $\delta=0$ so that only the condition $\sum_{i=1}^{t} d_{i}-$ $\sum_{i=1}^{n+t} \alpha_{i}=0$ is not solved by the above result. In particular, it follows from work of Kei-ichi Watanabe that if $R$ has an isolated rational singularity, then $R$ has $F$-pure type. The converse is also "almost true" with the only exception being the case where $\sum_{i=1}^{t} d_{i}-\sum_{i=1}^{n+t} \alpha_{i}=0$.

In proving this criterion, a weak but more stable form of $F$-purity, called $F$-contractedness, is defined and explored. $R$ is $F$-contracted (in characteristic $p>0$ ) if every system of parameters for $m$ is contracted with respect to the Frobenius map $F: R \rightarrow R$. Just as for $F$-purity, the notion of $F$-contracted type is defined in characteristic 0 by reduction to characteristic $p$. The two notions of $F$-pure (type) and $F$-contracted (type) coincide when $R$ is Gorenstein; whence, in particular, when $R$ is a complete intersection ring.
\end{abstract}

0. Introduction. Let $R$ be a ring of characteristic $p$ and let ${ }^{1} R$ denote the ring $R$ viewed as an $R$-module via the Frobenius map $F(r)=r^{p} . R$ is $F$-pure if for every $R$-module $M, 0 \rightarrow R \otimes M \rightarrow{ }^{1} R \otimes M$ is exact. A notion of $F$-pure type can then be defined in characteristic 0 by reduction to characteristic $p$.

The concept of $F$-purity was suggested originally by the Hochster-Roberts proof that the ring of invariants of a linearly reductive affine linear group acting on a regular ring is Cohen-Macaulay [4]. The same two authors later demonstrated that $F$-purity measures "nice" singularities in the sense that it implies much simplification (and vanishing) in the computation of local cohomology [5].

The problem of identifying those rings which are F-pure (type) is quite difficult. The following special cases are known:

(1) The invariant ring of a reductive linear group acting on a regular ring is $F$-pure (type) [5].

Received by the editors October 8, 1985.

1980 Mathematics Subject Classification (1985 Revision). Primary 13 H99.

The author wishes to thank the Research Council of the University of Missouri for a research grant which partially supported this project. 
(2) If $K$ is a field of characteristic 0 and $\mathscr{M}$ is a semigroup of monomials, then $K[\mathscr{M}]$ is $F$-pure type if and only if $\mathscr{M}$ is seminormal [5].

(3) The ring $S / I$, where $I$ is generated by square-free monomials, is $F$-pure (type) [5].

(4) If $(R, m, K)$ is a 1-dimensional local Noetherian ring of characteristic $p$ with $K \subset r$ an algebraically closed field and if ${ }^{1} R$ is a finite $R$-module, then $R$ is $F$-pure if and only if the $m$-adic completion of $R$ is isomorphic to

$$
K\left[\left[X_{1}, \ldots, X_{r}\right]\right] /\left(X_{i} X_{j} \mid 1 \leqslant i<j \leqslant r\right) \quad[3] .
$$

(5) Gorenstein square-free Hodge algebras are $F$-pure.

(6) The ring $R=K\left[X_{1}, \ldots, X_{n}\right] /(G)$, where $G$ is a quasihomogeneous polynomial with an isolated singularity at the origin, has $F$-pure type if $\operatorname{deg}(G)<n$. $R$ does not have $F$-pure type if $\operatorname{deg}(G)>n[2]$.

The purpose of this paper is to generalize result (6) to complete intersection rings and, at the same time, drop the isolated singularity requirement. We prove the following theorem.

TheOREM (HOMOGENEOUS VERSION). Let $S=K\left[X_{1}, \ldots, X_{n+t}\right]$ where $K$ is a field of characteristic 0 . Let $I=\left(G_{1}, \ldots, G_{t}\right)$ be a homogeneous complete intersection ideal in $S$ (i.e. $\left\{G_{1}, \ldots, G_{t}\right\}$ is a regular sequence in $S$ ). Denote $\sum_{i=1}^{t} \operatorname{deg}\left(G_{i}\right)=d$. Let $R=S / I$ and let $J$ be the radical ideal which determines the singular locus of $R$. Let $\delta$ be the length of the longest $R$-regular sequence in $J$. Then:

(1) $R$ has $F$-pure type if $d<\delta$.

(2) $R$ does not have F-pure type if $d>n$.

For a more precise statement of the main results, see Theorems 2.1 and 2.8 .

Kei-ichi Watanabe has proven that if $R$ as above has, in addition, an isolated singularity at $\mu=\left(x_{1}, \ldots, x_{n+t}\right)$. (Here, $x_{i}$ denotes the image of $X_{i}$ in $S / I$ ), then:

(1) $R$ has a rational singularity if $d<n$.

(2) $R$ does not have a rational singularity if $d \geqslant n[8]$.

Since $\delta$, as defined above, is $n$ when $R$ has an isolated singularity, our main theorem provides new evidence of the striking coincidence (in the known examples) between the notions of $F$-pure type and rational singularity.

\section{Definitions and preliminaries.}

Definition. Let $E \rightarrow E^{\prime}$ be an injective homomorphism of modules over a fixed base ring $R$. $E \rightarrow E^{\prime}$ is pure if, for every $R$-module $M, 0 \rightarrow E \otimes M \rightarrow E^{\prime} \otimes M$ is exact.

The existence of a splitting map from $E^{\prime}$ back to $E$ (which insures left exactness of tensor) would, of course, imply that $E \rightarrow E^{\prime}$ is pure. The converse is false. However, the reader may quite reasonably use the more familiar concept of "split" extensions as a source of intuition about pure extensions. In most circumstances, the two notions are equivalent. To be precise, we recall the following observations:

Lemma 1.1 [5, COROllary 5.2]. Let $R$ be a Noetherian subring of $S$. Then $R$ is a pure subring of $S$ if and only if $R$ is a direct summand, as an $R$-module, of every finitely generated $R$ submodule of $S$ which contains it. In particular, if $S$ is module finite over $R$, then $R \rightarrow S$ is pure if and only if it is split. 
LeMmA 1.2 [2, Lemma 1.2]. Let $(R, m)$ be a complete local ring and $M$ an $R$-module. Let $E$ be the injective hull of $R / m$ [7]. Then the following are eqivalent:

1. $R \stackrel{\alpha}{\rightarrow} M$ is split.

2. $R \stackrel{\alpha}{\rightarrow} M$ is pure.

3. $E \stackrel{\alpha}{\rightarrow} M \otimes E$ is injective.

Note that if $R \rightarrow M$ is a pure $R$-module map and if $I$ is an ideal in $R$, then $I M \cap R=I$; consequently, $I$ is a contracted ideal with respect to this map. For maps of the special type $R \rightarrow M$, Hochster defines cyclic purity to be the condition that every ideal is contracted and shows that under relatively mild assumptions about $R$, cyclic purity is equivalent to purity [6]. We weaken this condition somewhat.

Definition. Let $(R, \mu)$ be a local ring and $R \stackrel{\alpha}{\rightarrow} M$ be a map of $R$-modules. The map $\alpha$ is contracted if every ideal $I$ which is generated by a system of parameters for $\mu$ satisfies $I M \cap R=I$. If $R$ is not local, $R \rightarrow M$ is contracted provided that $R_{\mu} \rightarrow M_{\mu}$ is contracted for every $\mu \in \max \operatorname{Spec}(R)$.

One immediate consequence of the map $R \stackrel{\alpha}{\rightarrow} M$ being either pure or contracted is that it is injective. For purity, this follows from the definition simply by tensoring with $R$. For contractedness, note that if $\alpha(r)=0$, then certainly $\alpha(r) \in I M$ for every ideal $I$ which is generated by a system of parameters for some maximal ideal $\mu$ of $R$. The contractedness hypothesis then guarantees that $r$ lies in the intersection of all ideals of $R$ which are generated by systems of parameters. But this intersection is well known to be 0 .

If $R$ is a $K$-algebra where $K$ is a field of characteristic $p$, we denote by $F$ the Frobenius homomorphism, $F(r)=r^{p}$.

DEFINition. If $M$ is an $R$-module ( $R$ has characteristic $p$ ), ${ }^{1} M$ will denote the group $M$ viewed as an $R$-module via $r \cdot m=r^{p} m . R \stackrel{F}{\rightarrow}{ }^{1} R$ is therefore an $R$-module homomorphism.

Definition. $R$ is $F$-pure (respectively, $F$-contracted if $R \stackrel{F}{\rightarrow}{ }^{1} R$ is a pure (respectively, contracted) map.

In particular, for an $F$-pure or $F$-contracted ring, the Frobenius map must be injective; whence, $R$ has no nilpotents (reduced). When $R$ is reduced, there is a natural identification of maps:

(1) $R \stackrel{F}{\rightarrow}{ }^{1} R$.

(2) $R \rightarrow R^{1 / p}$ where $R^{1 / p}$ denotes the ring of $p$ th roots of elements in $R$.

(3) $R^{p} \rightarrow R$ where $R^{p}$ denotes the ring of $p$ th powers of elements in $R$.

To connect the notions of $F$-contractedness and $F$-purity with Watanabe's criterion for a rational singularity, we need to briefly review the construction of graded local cohomology. Actually the case of $H_{I}^{n}(R)$, where $I$ is the radical of an ideal generated by a regular sequence of length $n$, is the only one we will need for this paper, and it can be described in more down to earth terms than the other local cohomology modules. 
Let $R$ be a ring and $m$ a maximal ideal in $R$. Let $\bar{f}=\left\{f_{1}, \ldots, f_{n}\right\}$ be a finite set of elements in $m$ and let $M$ be any $R$-module. Recall that the Koszul homology $K_{*}(\bar{f} ; M)$ is the tensor product of complexes $\left(\otimes_{i=1}^{n} R \stackrel{f_{i}}{\rightarrow} R\right) \otimes_{R} M$, and the Koszul cohomology is given by $K^{*}(\bar{f}, M)=\operatorname{Hom}_{R}\left(K_{*}(\bar{f}, R), R\right) \otimes_{R} M$. Thus, the $n$th Koszul cohomology, $H^{n}\left(K^{*}(\bar{f} ; M)\right)$, is just $M /\left(f_{1}, \ldots, f_{n}\right) M$. Let $\bar{f}^{e}=$ $\left\{f_{1}^{e}, \ldots, f_{n}^{e}\right\}$. The $i$ th local cohomology, $H_{I}^{i}(M)$ is defined to be $\lim _{e} H^{i}\left(K^{*}\left(\bar{f}^{e} ; M\right)\right)$ where $I$ is the ideal generated by $\left\{f_{1}, \ldots, f_{t}\right\}$ (or the radical thereof). The direct limit maps $H^{i}\left(K^{*}\left(\bar{f}^{e} ; M\right)\right) \rightarrow H^{i}\left(K^{*}\left(\bar{f}^{e+1} ; M\right)\right)$ are induced by

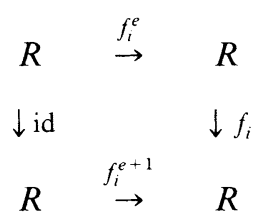

In particular, $H_{I}^{n}(M)$ is just $\lim _{e} M /\left(f_{1}^{e}, \ldots, f_{n}^{e}\right) M$ with direct limit maps being

$$
M /\left(f_{1}^{e}, \ldots, f_{n}^{e}\right) M \stackrel{\prod_{i=1}^{n} f_{i}}{\rightarrow} M /\left(f_{1}^{e+1}, \ldots, f_{n}^{e+1}\right) M .
$$

For a graded module $M,[M]_{t}$ will denote its $t$ h graded piece. In this paper, $R$ will always be a special graded $K$-algebra, by which we mean that $R$ is nonnegatively graded ring with $[R]_{0}=K$, a field. the irrelevant maximal ideal $\sum_{i=1}^{\infty}[R]_{i}$ will be denoted $\mu$. Note carefully that we do not require $\mu$ to be generated by $[R]_{1}$. If $\bar{f}=\left\{f_{1}, \ldots, f_{n}\right\}$, each $f_{i}$ is homogeneous of degree $d_{i}$, and $M$ is a graded $R$-module, then $H_{I}^{i}(M)$ naturally acquires a grading (in fact, independent of the choice of generators for the homogeneous ideal $I)$. Recall that $M(d)$ denotes the graded module $m$ with a shifted grading $[M(d)]_{t}=[M]_{d+t}$. Using this device, $R \stackrel{f_{i}}{\rightarrow} R\left(d_{i}\right)$ is a degree preserving $R$-module homomorphism, and both $\otimes_{i=1}^{n}\left(R \stackrel{f_{i}}{\rightarrow} R\left(d_{i}\right)\right) \otimes M$ $=K_{*}(\bar{f} ; M)$ and $\operatorname{Hom}_{R}\left(K_{*}(\bar{f} ; M), R\right)=K^{*}(\bar{f}, M)$ are complexes of graded modules and degree preserving maps. The homologies of such complexes are, of course, graded. In particular, since $R\left(t_{1}\right) \otimes R\left(t_{2}\right) \simeq R\left(t_{1}+t_{2}\right)$ as graded modules, $H^{n}\left(K^{*}(\bar{f} ; M)\right) \simeq(M /(\bar{f}) M)(d)$ as graded modules $\left(d=\sum_{i=1}^{n} \operatorname{deg}\left(f_{i}\right)\right)$. Thus, $H_{I}^{n}(M) \simeq \lim _{e}\left(M /\left(\bar{f}^{e}\right) M\right)(d e)$ which has a well-defined grading because the direct limit maps are degree preserving.

We now specialize to the case where $M={ }^{1} R$ (graded just as $R$ is). If $s \in{ }^{1} R$ and $f \in R$, then $f \cdot s=f^{p} s$ as an element of ${ }^{1} R$. Thus,

$$
H_{I}^{n}\left({ }^{1} R\right) \simeq \lim _{\rightarrow} \frac{{ }^{1} R}{\left(\bar{f}^{e}\right) \cdot{ }^{1} R}=\lim _{\rightarrow} \frac{R}{\left(\bar{f}^{p e}\right) R}
$$

and the direct limit maps consist of multiplication by $\prod_{i=1}^{n} f_{i}$ as $R$-modules; whence, multiplication by $\prod_{i=1}^{n} f_{i}^{p}$ as ${ }^{1} R$-modules. Ignoring the $R$-module structure, we see that the direct limit sequence for $H_{I}^{n}\left({ }^{1} R\right)$ is precisely that subsequence of the direct 
limit sequence for $H_{I}^{n}(R)$ consisting of every $p$ th group in the sequence (with the same maps!). It follows that $H_{I}^{n}\left({ }^{1} R\right)=H_{I}^{n}(R)$ as groups, or more precisely, $H_{I}^{n}\left({ }^{1} R\right)$ ${ }^{1}\left(H_{I}^{n}(R)\right) \simeq H_{I}^{n}(R) \otimes{ }^{1} R$ as $R$-modules. (Of course, the same is true for every $d$, $H_{I}^{d}\left({ }^{1} R\right)={ }^{1}\left(H_{I}^{d}(R)\right) \simeq H_{I}^{d}(R) \otimes{ }^{1} R$, but we will not use this fact for $d<n$.) Since $F$ : $R \rightarrow{ }^{1} R$ is an $R$-module homomorphism, it induces a homomorphism $F: H_{I}^{n}(R) \rightarrow$ $H_{I}^{n}\left({ }^{1} R\right)$. This can be obtained by applying $\otimes_{R} H_{I}^{n}(R)$ to $R \stackrel{F}{\rightarrow}{ }^{1} R$ or, more explicitly, by taking the direct limit of the induced maps from $H^{n}\left(K^{*}(\bar{f} ; R)\right)$ to $H^{n}\left(K^{*}\left(\bar{f} ;{ }^{1} R\right)\right)$. That is, $F: H_{I}^{n}(R) \rightarrow H_{I}^{n}\left({ }^{1} R\right)$ is the direct limit of the maps $R /\left(\bar{f}^{e}\right) R$ $\stackrel{F}{\rightarrow}{ }^{1}\left[R /\left(\bar{f}^{p e}\right) R\right]$ where, for $\bar{r}$ in $R /\left(\bar{f}^{e}\right) R, F(\bar{r})=\bar{r}^{p}$ in ${ }^{1}\left[R /\left(\bar{f}^{p e}\right) R\right]$. To understand the grade of this "Frobenius" map, we put in the appropriate twists to get

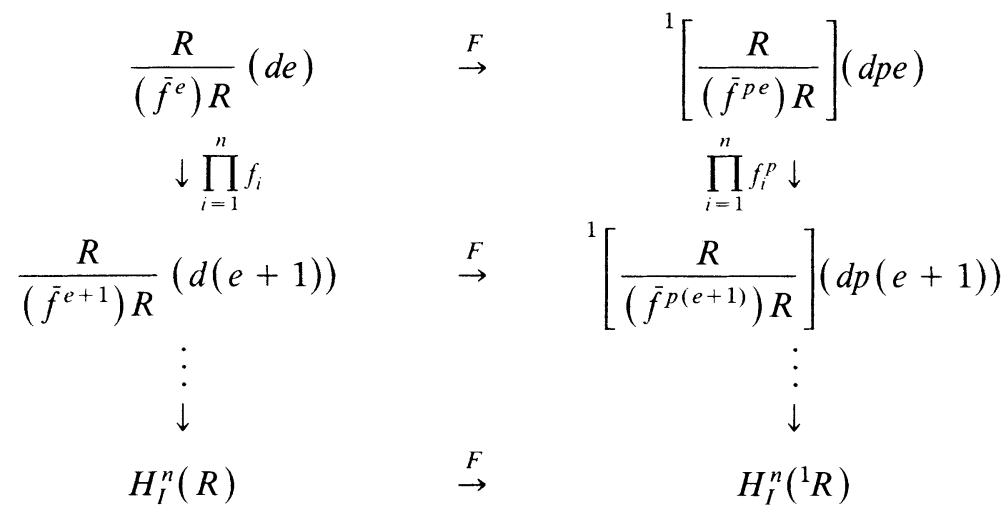

If $\alpha \in\left[H_{I}^{n}(R)\right]_{t}, \alpha$ can be represented as $\bar{r} \in\left[\left(R /\left(\bar{f}^{e}\right) R\right)(d e)\right]_{t}$ for some $e$ and $r$ can be chosen to be homogeneous in $R$ of degree $t+d e$. It follows that $F(\alpha)$ is represented by $\bar{r}^{p}$ in $R(d p e) /\left(\bar{f}^{p e}\right) R$ and, therefore, has degree $p t$ in $H_{I}^{n}(R)$. But, as already pointed out, $H_{I}^{n}\left({ }^{1} R\right)=H_{I}^{n}(R)$ as graded groups. In short, the Frobenius map induces a group homomorphism from $H_{I}^{n}(R)$ to itself which multiplies degrees by $p$. The same fact is true for $H_{I}^{d}(R)$ for $d<n$, a fact which was used by Hochster and Roberts to simplify the computation of local cohomology for $F$-pure rings.

Definition. Let $R$ be a special graded $K$-algebra with irrelevant ideal $\mu$ of dimension $n$. Then $a(R)=\max \left\{t \mid\left[H_{\mu}^{n}(R)\right]_{t} \neq 0\right\}$. That is, $a(R)$ is the largest nonvanishing graded piece of local cohomology.

DeFinition. A special graded $K$-algebra $R$ of dimension $n$ is a complete intersection if $R \simeq K\left[X_{1}, \ldots, X_{n+t}\right] /\left(G_{1}, \ldots, G_{t}\right)$ as a graded ring. (The $X_{i}$ 's are indeterminates of degree $\geqslant 1$.) $\left\{G_{1}, \ldots, G_{t}\right\}$ must therefore form a regular sequence, and the ideal they generate is called a complete intersection ideal.

Definition. If $(R, \mu)$ is a 0 -dimensional local ring, the socle of $R$ is the ideal $(0: \mu)=\{x \in R \mid x \mu=0\}$.

REMARKS 1.3. (1) For a Noetherian ring, each of its local cohomology modules satisfy DCC [7]. But, for each $N \in Z, \Sigma_{t>N}\left[H_{\mu}^{i}(R)\right]_{t}$ is a submodule of $H_{\mu}^{i}(R)$. Therefore, there exists $N$ such that, for every $t>N,\left[H_{\mu}^{i}(R)\right]_{t}=0$. This implies that $a(R)$ is finite.

(2) The socle of a 0 -dimensional graded ring is a homogeneous ideal. 
(3) If $R$ is an $n$-dimensional Cohen-Macaulay ring with a maximal ideal $\mu$ and $\bar{y}=\left\{y_{1}, \ldots, y_{n}\right\}$ is a system of parameters for $\mu$, then the direct limit system $\underset{\lim }{\rightarrow}\left(R /\left(\bar{y}^{e}\right) R\right)$ is injective, because, $\left(\prod_{i=1}^{n} y_{i}\right) h \in \bar{y}^{e+1}$ implies $h \in \bar{y}^{e}$ since $\left\{\overrightarrow{y_{1}}, \ldots, y_{n}\right\}$ is a regular sequence. Consequently, any homogeneous element of $H_{\mu}^{n}(R)$ having degree equal to $a(R)$ can always be represented by a homogeneous element of the socle of $R /\left(\bar{y}^{e}\right) R$ for some $e$.

(4) For a polynomial ring $S=K\left[X_{1}, \ldots, X_{n}\right], a(S)=-\sum_{i=1}^{n} \operatorname{deg}\left(X_{i}\right)$. To see this, write $H_{\mu}^{n}(S)=\lim _{\rightarrow}\left(S /\left(\bar{X}^{e}\right) S\right)(\alpha e)$ where $\bar{X}=\left\{X_{1}, \ldots, X_{n}\right\}$ and $\alpha=\sum \operatorname{deg}\left(X_{i}\right)$. For each $e$, the socle of $\left(S /\left(\bar{X}^{e}\right) S\right)(\alpha e)$ is obviously generated by $\prod_{i=1}^{n} X_{i}^{e-1}$ which has degree $\alpha(e-1)-\alpha e=-\alpha$. Thus, by the remark above, $a(S)=-\alpha$.

(5) If $R$ is a special graded Cohen-Macaulay $K$-algebra with irrelevant ideal $\mu$ and if $y$ is a homogeneous nonzero divisor in $R$, then $a(R /(y))=a(R)+\operatorname{deg}(y)$. Once we verify this fact, it follows that if $R=K\left[X_{1}, \ldots, X_{n+t}\right] /\left(G_{1}, \ldots, G_{t}\right)$ is a complete intersection ring with each $G_{i}$ being a homogeneous polynomial of degree $d_{i}$ then $a(R)=d-\alpha$ where $d=\sum_{i=1}^{t} d_{i}$ and $\alpha=\sum_{i=1}^{n+t} \operatorname{deg}\left(X_{i}\right)$.

That $a(R /(y))=a(R)+\operatorname{deg}(y)$ is an easy consequence of the long exact sequence of local cohomology. If $\operatorname{dim}(R)=n$ and we use ${ }^{-}$to denote reduction modulo $(y)$, then we have a long exact sequence of graded modules:

$$
\cdots \rightarrow H_{\mu}^{n-1}(R) \rightarrow H_{\mu}^{n-1}(\bar{R}) \stackrel{\delta}{\rightarrow} H_{\mu}^{n}(R) \stackrel{y}{\rightarrow} H_{\mu}^{n}(R) \rightarrow 0 .
$$

Obviously, multiplication by $y$ increases degrees by $\operatorname{deg}(y)$. And, it is not difficult to check that the boundary map $\delta$ decreases degrees by $\operatorname{deg}(y)$. Since $R$ is CohenMacaulay, $H_{\mu}^{n-1}(R)=0$. Thus, the long exact sequence above is actually a short exact sequence with graded maps. Taking graded pieces of this short exact sequence, we get

$$
0 \rightarrow\left[H_{\mu}^{n-1}(\bar{R})\right]_{d+\operatorname{deg}(y)} \stackrel{\delta}{\rightarrow}\left[H_{\mu}^{n}(R)\right]_{d} \stackrel{y}{\rightarrow}\left[H_{\mu}^{n}(R)\right]_{d+\operatorname{deg}(y)} \rightarrow 0 .
$$

Note that $H_{\mu}^{n-1}(\bar{R})$ is precisely the right cohomology module in which to compute $a(\bar{R})$ because $\operatorname{dim}(\bar{R})=n-1$ and $H_{\mu}^{n-1}(\bar{R})$ can be identified with $H_{\mu \bar{R}}^{n-1}(\bar{R})=$ $H_{\bar{\mu}}^{n-1}(\bar{R})$. Now, if we let $d=a(R)$ in the short exact sequence, we see that $\delta$ becomes an isomorphism $(\operatorname{deg}(y) \geqslant 1$ by hypothesis) and, consequently $\left[H_{\mu}^{n-1}(\bar{R})\right]_{a(R)+\operatorname{deg}(y)} \neq 0$. If, on the other hand, we assume $d>a(R)$, then $\delta$ injects $H_{\mu}^{n-1}(\bar{R})$ into 0 and, consequently, $\left[H_{\mu}^{n-1}(\bar{R})\right]_{d+\operatorname{deg}(y)}=0$ for all $d>a(R)$. Thus, $a(R /(y))=a(R)+\operatorname{deg}(y)$ as desired.

The connections between the Frobenius action on local cohomology and the property of being either $F$-pure or $F$-contracted have been partially discussed in $[\mathbf{2}, \mathbf{5}$, and 6]. Restricting our consideration to Cohen-Macaulay rings, we can extend and consolidate these ideas in Proposition 1.4 and Corollary 1.5.

Proposition 1.4. For a Cohen-Macaulay local ring $(R, \mu)$, the following are equivalent:

(1) The map from $H_{\mu}^{n}(R)$ to $H_{\mu}^{n}\left({ }^{1} R\right)$, induced by the Frobenius map from $R$ to ${ }^{1} R$, is injective. 
(2) $R$ is F-contracted.

(3) There exists a system of parameters for $\mu$ which is contracted with respect to the Frobenius map from $R$ to ${ }^{1} R$.

Proof. Note that an ideal $\left(z_{1}, \ldots, z_{s}\right)$ is contracted with respect to the Frobenius if and only if $F: R /\left(z_{1}, \ldots, z_{s}\right) \rightarrow^{1}\left[R /\left(z_{1}^{p}, \ldots, z_{s}^{p}\right)\right]$ is injective. To prove $(1) \Rightarrow(2)$, then, we must show that for any system of parameters $\bar{y}=\left\{y_{1}, \ldots, y_{n}\right\}, F$ : $R /(\bar{y}) \rightarrow R /\left(\bar{y}^{p}\right)$ is injective. (Here $\bar{y}^{e}=\left\{y_{1}^{e}, \ldots, y_{n}^{e}\right\}$.) Let $\alpha$ be an element of the kernel of this map. Since $F: H_{\mu}^{n}(R) \rightarrow H_{\mu}^{n}\left({ }^{1} R\right)$ is just the direct limit of maps $F$ : $R /\left(\bar{y}^{e}\right) \rightarrow R /\left(\bar{y}^{p e}\right)$, the injective hypothesis on local cohomology implies that $\alpha$ is equivalent to 0 as a representative of local cohomology. But, since the direct limit system, $H_{\mu}^{n}(R)=\lim _{e}\left(R /\left(\bar{y}^{e}\right)\right)$ is injective (because $R$ is Cohen-Macaulay) $\alpha$ must already be 0 in $R /(\bar{y})$.

$(2) \Rightarrow(3)$ is true by definition.

To prove $(3) \Rightarrow(1)$, recall that, denoting $K=R / \mu$, the Cohen-Macaulay type of $R$ is given by

$$
\begin{aligned}
d & =\operatorname{dim}_{K}\left(\operatorname{Ext}_{R}^{n}(K, R)\right)=\operatorname{dim}_{K}\left(\operatorname{Hom}_{R}\left(K, \frac{R}{\left(z_{1}, \ldots, z_{n}\right)}\right)\right) \\
& =\operatorname{dim}_{K} \frac{\left(z_{1}, \ldots, z_{n}\right):(\mu)}{\left(z_{1}, \ldots, z_{n}\right)}
\end{aligned}
$$

independent of the choice of system of parameters $\left(z_{1}, \ldots, z_{n}\right)$ for $\mu$. Let $\bar{y}=$ $\left(y_{1}, \ldots, y_{n}\right)$ be a given system of parameters which is contracted with respect to the Frobenius map. Then $\bar{y}^{e}$ is a system of parameters (i.e. maximal regular sequence) in $\mu$ for every $e \geqslant 1$. Note that the vector space $\left(\left(\bar{y}^{e}\right):(\mu)\right) /\left(\bar{y}^{e}\right)$, whose dimension $d$ is the Cohen-Macaulay type of $R$, is just the socle of $R /\left(\bar{y}^{e}\right)$. Moreover, the direct limit map from $R /\left(\bar{y}^{e}\right)$ to $R /\left(\bar{y}^{e+1}\right)$, multiplication by $\prod_{i=1}^{n} y_{i}$, induces a vector space homomorphism on the respective socles-well defined because $\alpha \in\left(\left(\bar{y}^{e}\right): \mu\right)$ $\Rightarrow\left(\prod_{i=1}^{n} y_{i}\right) \alpha \in\left(\left(\bar{y}^{e+1}\right): \mu\right)$ and $\alpha \in\left(\bar{y}^{e}\right) \Rightarrow\left(\Pi_{i=1}^{n} y_{i}\right) \alpha \in \bar{y}^{e+1}$. If $\alpha \in R$ is such that $\bar{\alpha} \in \operatorname{socle}\left(R /\left(\bar{y}^{e}\right)\right)$ and if $\left(\prod_{i=1}^{n} y_{i}\right)(\bar{\alpha}) \equiv 0$ in $R /\left(\bar{y}^{e+1}\right)$, then $\alpha \in$ $\left(\bar{y}^{e+1}\right):\left(\prod_{i=1}^{n} y_{i}\right)=\left(\bar{y}^{e}\right)$ and therefore $\bar{\alpha}=0$ in the socle of $R /\left(\bar{y}^{e}\right)$. Thus, multiplication by $\prod_{i=1}^{n} y_{i}$ induces an injective map on the respective socles. But since the dimension of the socle of $R /\left(\bar{y}^{e+1}\right)$, equals the dimension of the socle of $R /\left(\bar{y}^{e}\right)$ as a $K$-vector space, multiplication by $\prod_{i=1}^{n} y_{i}$ must induce an isomorphism. In other words, for every $e \geqslant 1$, the socle of $R /\left(\bar{y}^{e}\right)$ is isomorphically identified with the socle of $R /(\bar{y})$ via the direct limit maps for $H_{\mu}^{n}(R)$. Now, if $F: H_{\mu}^{n}(R) \rightarrow H_{\mu}^{n}\left({ }^{1} R\right)$ were not injective, there would exist $0 \neq \gamma \in H_{\mu}^{n}(R)$, represented by an element of the socle of $R /\left(\bar{y}^{e}\right)$ for some $e$, such that $F(\gamma)=0$. But, by the above isomorphism $\gamma$ can in fact be represented by some $\alpha$ in the socle of $R /(\bar{y})$. The assumption that $\left(y_{1}, \ldots, y_{n}\right)$ is contracted would then imply that $F(\alpha) \neq 0$ in $R /\left(\bar{y}^{p}\right)$. Hence, $F(\alpha) \neq 0$ in $H_{\mu}^{n}\left({ }^{1} R\right)$, a contradiction.

For a Gorenstein local ring $(R, \mu)$ of dimension $n$, it is a standard fact from local duality theory that $H_{\mu}^{n}(R)$ is isomorphic to $E$, the injective hull of $R / \mu$ [7]. 
Hochster and Roberts proved that $R$ is $F$-pure if and only if $E \rightarrow E \otimes{ }^{1} R$ is injective [5]. Hence

Corollary 1.5. Let $(R, \mu)$ be a local Gorenstein ring (e.g. $R$ is a complete intersection ring). The following are equivalent:

(1) $R$ is F-pure.

(2) $R$ is F-contracted.

(3) There exists a system of parameters which is contracted with respect to the Frobenius map.

(4) $H_{\mu}^{n}(R) \stackrel{F}{\rightarrow} H_{\mu}^{n}\left({ }^{1} R\right)$ is injective.

The reader should be cautioned that, whereas conditions (2), (3), and (4) are equivalent for Cohen-Macaulay rings and condition (1) implies each of the others, (1) is not equivalent to (2), (3), and (4) in general [2]. Moreover, if $R$ is not Cohen-Macaulay, the equivalences between (2), (3), and (4) also break down.

$F$-purity is a characteristic $p$ notion, but our primary interest is in rings of characteristic 0 . To pass from characteristic 0 to characteristic $p$ :

DEFINITION. Let $W$ be a property defined for rings of characteristic $p$.

(1) Let $R=A\left[X_{1}, \ldots, X_{n}\right] /\left(F_{1}, \ldots, F_{t}\right)$ where $A$ is a ring of mixed characteristic. We define a notion of $W$-type which is stable under localization at infinitely many elements of $A$. Let $Y$ be the maximal spectrum of $A$ and denote $K_{m}=A / m$ for $m \in Y . R$ has open (respectively, dense) $W$-type if there is a nonempty Zariski open (respectively, dense) subset $U \subset Y$ such that for every $m \in U, R \otimes_{A} K_{m}$ satisfies $W$.

(2) Let $T=K\left[X_{1}, \ldots, X_{n}\right] /\left(F_{1}, \ldots, F_{t}\right)$ where $K$ is a field of characteristic $0 . T$ has $W$-type if there exists a ring $A \subset K$ of mixed characteristic such that, for each $1 \leqslant i \leqslant t, F_{i} \in A\left[X_{1}, \ldots, X_{n}\right]$ and $A\left[X_{1}, \ldots, X_{n}\right] /\left(F_{1}, \ldots, F_{t}\right)$ has $W$-type.

At the beginning of $\S 4$ of the Hochster-Roberts paper [5], $F$-pure type is defined in 16 variations. The above definition corresponds to "having a presentation of $F$-pure type." By [2, Proposition 1.11], this is equivalent to "having a presentation of perfect $F$-pure type," so that we can replace $K_{m}$ in the definition by its perfect closure $\bar{K}_{m}\left(a^{P} \in \bar{K}_{m} \Rightarrow a \in \bar{K}_{m}\right)$. If $R$ is the ring in the above definition and $S=R \otimes_{A} \bar{K}_{m}$, then ${ }^{1} S$ is module finite over $S$ [2, Lemma 1.5]; so, $S \rightarrow{ }^{1} S$ is pure if and only if it splits (Lemma 1.1).

II. Complete intersection rings of $F$-pure type. We standardize notation throughout this section. $S=K\left[X_{1}, \ldots, X_{n+t}\right]$ will be a graded polynomial ring such that $\operatorname{deg}\left(X_{i}\right)>0$ for each $1 \leqslant i \leqslant n+t$. (Note that we do not require $\operatorname{deg}\left(X_{i}\right)=1$.) $R=S /\left(G_{1}, \ldots, G_{t}\right)$ will be a graded ring, graded consistently with the preassigned grading of $S$ (which may be nonstandard). Polynomials in $S$ will be represented by capital letters whereas equivalence classes of polynomials in $R$ will be represented by lowercase letters. $D_{i}\left(G_{j}\right)$ will denote the partial derivative of $G_{J}$ with respect to $X_{i}$. $J$ will denote the Jacobian ideal in $S$ which defines the singular locus of $S / I=R$. Recall that if $\left(G_{1}, \ldots, G_{t}\right)$ is a complete intersection ideal, then $J$ is the ideal generated by the $t \times t$ determinantal minors of the $(n+t) \times t$ matrix of 
partial derivatives $\left(D_{i}\left(G_{J}\right)\right)$. The characteristic $p$ version of the main theorem is as follows:

THEOREM 2.1. Let $S=K\left[X_{1}, \ldots, X_{n+t}\right]$ where $K$ is a field of characteristic $p$ and let $R=S /\left(G_{1}, \ldots, G_{t}\right)$ be a special graded complete intersection ring of dimension $n$ with irrelevant ideal $\mu=R_{+}$. Assume that $\left\{Y_{1}, \ldots, Y_{n}\right\}$ is a homogeneous regular sequence in $S$ whose image, $\left\{y_{1}, \ldots, y_{n}\right\}$ in $R$ forms a maximal (homogeneous) regular sequence. Further assume that $\left(Y_{1}, \ldots, Y_{s}\right)$ is contained in the Jacobian ideal $J$ for some $1 \leqslant s \leqslant n$. Then if $a(R)<-\sum_{i=s+1}^{n} \operatorname{deg}\left(Y_{i}\right)$, either $R$ is F-pure or $p<$ $\sum_{i=1}^{s} \operatorname{deg}\left(Y_{i}\right)$.

Proof. We induct on $t$, the height of the complete intersection ideal (If $t=0$, then $R=S$ is a polynomial ring which is easily seen to be $F$-pure.). To carry out this induction, we need to see that if $R=S /\left(G_{1}, \ldots, G_{t}\right)$ satisfies the hypotheses of the theorem, then so does $T=S /\left(G_{1}, \ldots, G_{t-1}\right)$. Since $R=T /\left(G_{t}\right)$ and $G_{t}$ is a nonzero divisor on $T$, it is clear that the images of $\left(Y_{1}, \ldots, Y_{n}, G_{t}\right)$ in $T$ form a maximal regular sequence in the irrelevant maximal ideal of $T$. It is also clear that

$$
I_{t-1}\left[\begin{array}{ccc}
D_{1}\left(G_{1}\right) & \cdots & D_{1}\left(G_{t-1}\right) \\
\vdots & & \vdots \\
D_{n+t}\left(G_{1}\right) & \cdots & D_{n+t}\left(G_{t-1}\right)
\end{array}\right] \supset I_{t}\left[\begin{array}{ccc}
D_{1}\left(G_{1}\right) & \ldots & D_{1}\left(G_{t}\right) \\
\vdots & & \vdots \\
D_{n+t}\left(G_{1}\right) & \ldots & D_{n+t}\left(G_{t}\right)
\end{array}\right]
$$

In other words, the Jacobian ideal associated to $T$ contains the one associated to $R$ which, in particular, contains $\left(Y_{1}, \ldots, Y_{s}\right)$. Since $a(R)=a\left(T /\left(G_{t}\right)\right)=a(T)+$ $\operatorname{deg}\left(G_{t}\right)$ [Remark 1.3] the fact that $a(R)<-\sum_{i=s+1}^{n} \operatorname{deg}\left(Y_{i}\right)$ implies that $a(T)<$ $-\sum_{i=s+1}^{n} \operatorname{deg}\left(Y_{i}\right)-\operatorname{deg}\left(G_{t}\right)$. Therefore, denoting $G_{t}=Y_{n+1}$, the images of $\left(Y_{1}, \ldots, Y_{n}, Y_{n+1}\right)$ in the special graded complete intersection ring $T$ form a maximal homogeneous regular sequence such that $\left(Y_{1}, \ldots, Y_{s}\right)$ is contained in the Jacobian ideal of $T$ and $a(T)<-\sum_{i=s+1}^{n+1} \operatorname{deg}\left(Y_{i}\right)$. That is, $T$ satisfies the "same" hypothesis as $R$.

The following ideals will be used frequently in the ensuing discussions:

(1) $I=\left(Y_{1}, \ldots, Y_{n}, G_{1}, \ldots, G_{t}\right)$.

(2) $\Gamma_{r}=\left(Y_{1}^{p}, \ldots, Y_{n}^{p}, G_{1}, \ldots, G_{t-1}, G_{t}^{r}\right)$ for $r \geqslant 1$. In particular, note that $R /\left(y_{1}^{p}, \ldots, y_{n}^{p}\right)=S / \Gamma_{1}$ and $R /\left(y_{1}, \ldots, y_{n}\right)=S / I$.

To prove the theorem, we must show that $a(R)<-\sum_{i=s+1}^{n} \operatorname{deg}\left(Y_{i}\right)$ and $R$ not $F$-pure together imply that $p<\sum_{i=1}^{s} \operatorname{deg}\left(Y_{i}\right)$. Since $R$ is Gorenstein, the $F$-contractedness test applied to any one system of parameters will suffice to determine $F$-purity (Proposition 1.4 and Corollary 1.5). Thus, if $R$ is not $F$-pure, $F$ : $\left(R /\left(y_{1}, \ldots, y_{n}\right)\right)(\alpha) \rightarrow\left(R /\left(y_{1}^{p}, \ldots, y_{n}^{p}\right)(p \alpha)\right.$ is not injective. (Here, $\alpha=\sum_{i=1}^{n} \operatorname{deg}\left(Y_{i}\right)$ and, as usual, the twists $(\alpha)$ and $(p \alpha)$ indicate the way in which $R /\left(y_{1}, \ldots, y_{n}\right)$ and $R /\left(y_{1}^{p}, \ldots, y_{n}^{p}\right)$ are embedded as graded submodules of $H_{\mu}^{n}(R)$ and $H_{\mu}^{n}\left({ }^{1} R\right)$ respectively.) It follows that there is a homogeneous $h \in R$ such that $0 \neq \bar{h} \in$ $R(\alpha) /\left(y_{1}, \ldots, y_{n}\right)$, but $\bar{h}^{p}=0$ in $R /\left(y_{1}^{p}, \ldots, y_{n}^{p}\right)$. Moreover $\bar{h} \neq 0$ in $H_{\mu}^{n}(R)$; therefore $\operatorname{deg}(h)-\alpha \leqslant a(R)<-\sum_{i=s+1}^{n} \operatorname{deg}\left(Y_{i}\right)$. Pulling back to $S$, there exists a 
homogeneous polynomial $H \in S$ such that

$$
\left\{\begin{array}{ll}
(1) & H^{p} \in \Gamma_{1} \\
\text { (2) } & H \notin I . \\
\text { (3) } & \operatorname{deg}(H)<\alpha-\sum_{i=s+1}^{n} \operatorname{deg}\left(Y_{i}\right)=\sum_{i=1}^{s} \operatorname{deg}\left(Y_{i}\right) .
\end{array}\right\}
$$

Since $\left\{H, Y_{1}, \ldots, Y_{n}, G_{1}, \ldots, G_{t}\right\}$ are all homogeneous polynomials, we may assume that the alleged expression of the form $H^{p}=A_{1} Y_{1}^{p}+\cdots+A_{n} Y_{n}^{p}+B_{1} G_{1}$ $+\cdots+B_{t} G_{t}$ is homogeneous (i.e. the degree of each term is the same, namely $p \operatorname{deg}(H))$. It would be convenient, but unjustifiable, to assume in the expression above that $B_{t} \notin \Gamma_{1}$. However, making judicious use of our induction hypothesis, we can reduce to a slightly more technical standard form of condition *. (The details of this reduction will be presented later.) The essence of our proof is the somewhat surprising fact that if $J \supset\left(Y_{1}, \ldots, Y_{s}\right)$ and if $H \in S$ satisfies

$$
* * \quad\left\{\begin{array}{ll}
(1) \quad H^{p}=A_{1} Y_{1}^{p}+\cdots+A_{n} Y_{n}^{p}+B_{1} G_{1}+\cdots+B_{t-1} G_{t-1}+B_{t} G_{t}^{r} \\
\\
\text { where this expression for } H^{p} \text { is homogeneous. } \\
(2) \quad B_{t} \notin \Gamma_{1} \text { and } p \text { does not divide } r . \\
(3) & \operatorname{deg}(H)<\sum_{i=1}^{s} \operatorname{deg}\left(Y_{i}\right) .
\end{array}\right\}
$$

then $P<\sum_{i=s+1}^{n} \operatorname{deg}\left(Y_{i}\right)$.

To demonstrate that $* * \Rightarrow p<\sum_{i=s+1}^{n} \operatorname{deg}\left(Y_{i}\right)$, let $D$ be any derivation from $S$ to $S$ and note that $D\left(H^{p}\right)=0$. Therefore, applying $D$ to $* *(1)$ yields

$$
0 \equiv B_{1} D\left(G_{1}\right)+\cdots+B_{t-1} D\left(G_{t-1}\right)+r B_{t} G_{t}^{r-1} D\left(G_{t}\right) \quad \text { (modulo } \Gamma_{r} \text { ). }
$$

In particular, if $1 \leqslant i_{1}<i_{2}<\cdots<i_{t} \leqslant n+t$ is a $t$-tuple of integers and $D_{i_{J}}=$ $\partial / \partial X_{i_{J}}$, we get a matrix equation

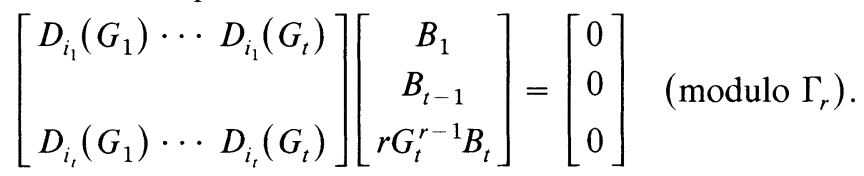

Multiplying by the cofactor transpose of the above matrix, denoting its determinant by $\Delta_{i_{1} \ldots i_{t}}$, and considering only the bottom equation that results, we see that $r G_{t}^{r-1} B_{t} \Delta_{i_{1} \cdots i_{t}} \equiv 0$ (modulo $\Gamma_{r}$ ). As $p$ does not divide $r, G_{t}^{r-1} B_{t} \Delta_{i_{1} \cdots i_{t}} \equiv 0$. But the Jacobian ideal $J$ is generated by the $\Delta$ 's ranging over all possible $t$-tuples; therefore, $G_{t}^{r-1} B_{t} \in\left(\Gamma_{r}: J\right)$. Since $J \supset\left(Y_{1}, \ldots, Y_{s}\right),\left(\Gamma_{r}: J\right) \subset\left(\Gamma_{r}:\left(Y_{1}, \ldots, Y_{s}\right)\right)$. This last ideal, being a colin of ideals generated by regular sequences, readily simplifies [1] to $\left(\prod_{i=1}^{s} Y_{i}^{p-1}, \Gamma_{r}\right)$. This means that $B_{t} G_{t}^{r-1} \in\left(\prod_{i=1}^{s} Y_{i}^{p-1}, \Gamma_{r}\right)$.

We next claim that $B_{t} \in\left(\prod_{i=1}^{s} Y_{i}^{p-1}, \Gamma_{1}\right) \backslash \Gamma_{1}$. By hypothesis $* *(2), B_{t} \notin \Gamma_{1}$ and, so, we need only show that $B_{t} \in\left(\Pi Y_{i}^{p-1}, \Gamma_{1}\right)$. Since

$$
\begin{aligned}
B_{t} G_{t}^{r-1} & \in\left(\prod_{i=1}^{s} Y_{i}^{p-1}, \Gamma_{r}\right) \subset \bigcap_{i=1}^{s}\left(Y_{1}^{p}, \ldots, Y_{i}^{p-1}, \ldots, Y_{n}^{p}, G_{1}, \ldots, G_{t-1}, G_{t}^{r}\right), \\
B_{t} & \in\left(\bigcap_{i=1}^{s}\left(Y_{1}^{p}, \ldots, Y_{i}^{p-1}, \ldots, Y_{n}^{p}, G_{1}, \ldots, G_{t-1}, G_{t}^{r}\right)\right):\left(G_{t}^{r-1}\right)
\end{aligned}
$$


which equals

$$
\bigcap_{i=1}^{s}\left(Y_{1}^{p}, \ldots, Y_{i}^{p-1}, \ldots, Y_{n}^{p}, G_{1}, \ldots, G_{t-1}, G_{t}^{r}\right):\left(G_{t}^{r-1}\right) .
$$

This last ideal is an intersection of ideals which are colins of regular sequences, and so we can simplify:

$$
B_{t} \in \bigcap_{i=1}^{s}\left(Y_{1}^{p}, \ldots, Y_{i}^{p-1}, \ldots, Y_{n}^{p}, G_{1}, \ldots, G_{t}\right) \subset\left(\Gamma_{1}\right):\left(Y_{1}, \ldots, Y_{s}\right) .
$$

But $\left(\Gamma_{1}\right):\left(Y_{1}, \ldots, Y_{s}\right)=\left(\prod_{i=1}^{s} Y_{i}^{p-1}, \Gamma_{1}\right)$ because it is, once again, a colin of regular sequences. Thus, $B_{t} \in\left(\Pi Y_{i}^{p-1}, \Gamma_{1}\right) \backslash \Gamma_{1}$ as claimed.

It follows that

$$
\operatorname{deg}\left(B_{t}\right) \geqslant \operatorname{deg}\left(\prod_{i=1}^{s} Y_{i}^{p-1}\right)=(p-1) \sum_{i=1}^{s} \operatorname{deg}\left(Y_{i}\right) .
$$

However, counting degrees in equation $* *(1)$ yields

$$
p \operatorname{deg}(H)=\operatorname{deg}\left(B_{t}\right)+r \operatorname{deg}\left(G_{t}\right) .
$$

Therefore $(p-1) \sum_{i=1}^{s} \operatorname{deg}\left(Y_{i}\right) \leqslant \operatorname{deg}\left(B_{t}\right)<p \operatorname{deg}(H)$. Solving for $p$, we get

$$
p\left[\sum_{i=1}^{s} \operatorname{deg}\left(Y_{i}\right)-\operatorname{deg}(H)\right]<\sum_{i=1}^{s} \operatorname{deg}\left(Y_{i}\right) .
$$

Since, by $* *(3), \operatorname{deg}(H)$ is strictly less than $\sum_{i=1}^{s} \operatorname{deg}\left(Y_{i}\right)$, it follows that $p<$ $\sum_{i=1}^{s} \operatorname{deg}\left(Y_{i}\right)$.

Summarizing, we have proven thus far that $* * \Rightarrow p<\sum_{i=1}^{s} \operatorname{deg}\left(Y_{i}\right)$, the $\operatorname{desired}$ conclusion for our theorem, and that $R$ not $F$-pure and $a(R)<-\sum_{i=s+1}^{n} \operatorname{deg}\left(Y_{i}\right)$ together imply $*$. It remains only to prove, given the induction hypothesis by which we can assume the theorem to be true for $T=S /\left(G_{1}, \ldots, G_{t-1}\right)$, that $* \Rightarrow * *$ or $p<\sum_{i=1}^{s} \operatorname{deg}\left(Y_{i}\right)$.

Note that the theorem being true for $T$ means that $T$ is either $F$-pure or $p<\sum_{i=1}^{s} \operatorname{deg}\left(Y_{i}\right)$; and, in the latter case, there is nothing left to prove. Therefore, we may assume that $T$ is $F$-pure. Again, applying the $F$-contractedness criterion (Corollary 1.5), to the particular system of parameters $\left(\bar{Y}_{1}, \ldots, \bar{Y}_{n}, \bar{G}_{t}\right)$ in $T$, the $F$-purity of $T$ translates into the condition that, for every $H \in S, H^{p} \in \Gamma_{p}=$ $\left(Y_{1}^{p}, \ldots, Y_{n}^{p}, G_{t}^{p}, G_{1}, \ldots, G_{t-1}\right)$ implies $H \in I=\left(Y_{n}, G_{t}, G_{1}, \ldots, G_{t-1}\right)$. But $H \in I$ contradicts $*$. Hence, the $F$-purity of $T$ in the presence of $*$ implies $H^{p} \notin \Gamma_{p}$.

Now, by hypothesis $*, H^{p} \in \Gamma_{1} \backslash \Gamma_{p}$. Therefore, there exists an integer $1 \leqslant r<p$ such that $H^{p} \in \Gamma_{r} \backslash \Gamma_{r+1}$. This means that we can write

$$
H^{p}=A_{1} Y_{1}^{p}+\cdots+A_{n} Y_{n}^{p}+B_{1} G_{1}+\cdots+B_{t-1} G_{t-1}+B_{t} G_{t}^{r},
$$

and we may assume this expression is homogeneous. It follows that $B_{t} \notin \Gamma_{1}$. For if $B_{t} \in \Gamma_{1}$, then $H^{p} \in \Gamma_{r+1}$, a contradiction. That

$$
H^{p}=A_{1} Y_{1}^{p}+\cdots+A_{n} Y_{n}^{p}+B_{1} G_{1}+\cdots+B_{t-1} G_{t-1}+B_{t} G_{t}^{r}
$$

is a homogeneous equation with $r$ strictly less than $p$ and $B_{t} \notin \Gamma_{1}$ guarantees that all of the conditions of $* *$ are satisfied. 
REMARK 2.2. Theorem 2.1 essentially says that if $a(R)$ is sufficiently small, $R$ should be $F$-pure. It is interesting to note the partial converse-if $R$ is $F$-pure, then $a(R) \leqslant 0$ [5]. This follows from the fact that $H_{\mu}^{n}(R)$ has DCC; whence, there exists $N$ such that $\left[H_{\mu}^{n}(R)\right]_{e}=0$ for every $e \geqslant N$. As pointed out in $\S 1$, (ignoring the funny $R$-module structure of ${ }^{1} H_{\mu}^{n} R$ ), we can think of $F$ as a homomorphism of graded groups which multiplies degrees by $p$. When $R$ is $F$-pure, this homomorphism is injective. But, then, iterating $F$ sufficiently, every positively graded piece of $H_{\mu}^{n}(R)$ can be embedded eventually into $0=\left[H_{\mu}^{n}(R)\right]_{e}$ for $e>N$.

ReMARK 2.3. The case where $a(R)$ is trapped between $-\sum_{i=s+1}^{n} \operatorname{deg}\left(Y_{i}\right)$ and 0 is more ambiguous and subtle. On the one hand, $R=K[X, Y] /\left(X^{2}+Y^{2}\right)$ is $F$-pure for all primes $p \neq 2$ because $\left(X^{2}+Y^{2}\right)^{p-1} \notin\left(X^{p}, Y^{p}\right) S$. (See [2, Theorem 1.12] for this criterion for F-purity.) Yet, $a(R)=\operatorname{deg}(X)+\operatorname{deg}(Y)-\operatorname{deg}\left(X^{2}+Y^{2}\right)=0$. On the other hand, $T=K\left[X_{1}, \ldots, X_{n}\right] /\left(X_{1}^{n}+X_{2}^{n}+\cdots+X_{n}^{n}\right)$ is $F$-pure if and only if $p \equiv 1$ modulo $n$; whence, the same is true for

$$
W_{k}=L\left[X_{1}, \ldots, X_{n}\right]\left[Z_{1}, \ldots, Z_{k}\right] /\left(X_{1}^{n}+\cdots+X_{n}^{n}\right) .
$$

Since the radical of the Jacobian ideal for $W_{k}$ contains precisely $\left(X_{1}, \ldots, X_{n}\right)$, the complementary regular sequence prescribed in Theorem 2.1 can be chosen to be $\left(Z_{1}, \ldots, Z_{k}\right)$. Thus, " $-\sum_{i=s+1}^{n} Y_{i}$ " in this example is $-\sum_{i=1}^{k} \operatorname{deg}\left(Z_{i}\right)=-k$. Also, $a\left(W_{k}\right)=-k=$ " $-\sum_{i=s+1}^{n} \operatorname{deg}\left(Y_{i}\right)$ ". Yet, if $n>2$, there are infinitely many primes for which $p \not \equiv 1$ modulo $n$; so, there does not exist an $N$ for which $p>N$ would imply $W_{k}$ is $F$-pure.

In some sense a result of the form $R$ is $F$-pure or $p<N$ (where $N$ is fixed independent of $p$ ) is automatically a theorem in characteristic 0 . We could state immediately that "complete intersection type" rings which have "isolated singularity type" and " $a(R)$ type" $<0$ have $F$-pure type. Of course such a result would be obscure (to the point of nonsense). We must therefore analyze more carefully the effect on these properties of the reduction process from characteristic 0 to characteristic $p$.

Again, in the hypotheses of the next several results, $S=K\left[X_{1}, \ldots, X_{n+t}\right]$ and $R=S /\left(G_{1}, \ldots, G_{t}\right)$. But, now, $K$ will be a field of characteristic 0 . If $A \subset K$ is any ring of mixed characteristic and $m$ is a maximal ideal in $A$, then $K_{m}$ will denote the field $A / m$ of characteristic $p$. We will denote $S_{m}=K_{m}\left[X_{1}, \ldots, X_{n+t}\right]$ and, where the notion makes sense, $R_{m}=K_{m}\left[X_{1}, \ldots, X_{n+t}\right] /\left(G_{1}, \ldots, G_{t}\right)$. Of course, this last definition of $R_{m}$ makes sense precisely when the polynomials $G_{1}, \ldots, G_{t}$ lie in the subring $A\left[X_{1}, \ldots, X_{n+t}\right] \subset K\left[X_{1}, \ldots, X_{n+t}\right]$, that is, precisely when all the coefficients of each of the $G_{i}$ 's lie in the coefficient subring $A \subset K$. Again, $\mu$ will denote the maximal ideal $\left(X_{1}, \ldots, X_{n}\right)$. The same symbol will be used (ambiguously) whether we are talking about the maximal ideal of $S, R, S_{m}$ or $R_{m}$. As usual, capital letters will denote polynomials in $S$ or $S_{m}$ whereas small letters will be used for their images in $R$ or $R_{m}$. "-" will be used to signify passage from $A\left[X_{1}, \ldots, X_{n+t}\right]$ to $K_{m}\left[X_{1}, \ldots, X_{n+t}\right]$. Finally $Z$ will denote the integers viewed as a subring of $K$. 
LeMma 2.4. Let $\left(H, F_{1}, \ldots, F_{d}\right) \in S$ satifsy $H \in\left(F_{1}, \ldots, F_{d}\right) S$ (respectively, $H \in$ $\left.\left(F_{1}, \ldots, F_{d}\right) S_{\mu}\right)$. Then there exists $A_{0}$ finitely generated over $Z$ with the property:

For every finitely generated Z-algebra $A$ between $A_{0}$ and $K,\left(H, F_{1}, \ldots, F_{d}\right) \subset$ $A\left[X_{1}, \ldots, X_{n+t}\right]$ and, there is a dense open set $U$ in $\operatorname{maxSpec}(A)$ such that for every $m \in U, \bar{H} \in\left(\bar{F}_{1}, \ldots, \bar{F}_{d}\right) S_{m}$ (respectively, $\left.\bar{H} \in\left(\bar{F}_{1}, \ldots, \bar{F}_{d}\right)\left(S_{m}\right)_{\mu}\right)$.

Proof. We will consider only the case $H \in\left(F_{1}, \ldots, F_{t}\right) S_{\mu}$. (The case $H \in$ $\left(F_{1}, \ldots, F_{d}\right) S$ follows trivially.) Let $H=\sum_{i=1}^{d} C_{i} F_{i} / B_{i}$ where $B_{i}, C_{i} \in S$ and $B_{i} \notin \mu$ (i.e. $B_{i}$ has a nonzero constant term). By adjoining to $Z$ all the coefficients from $K$ of each of the polynomials $\left\{H, F_{1}, \ldots, F_{d}, B_{1}, \ldots, B_{d}, C_{1}, \ldots, C_{d}\right\}$, we can construct $A_{0}$ so that each of these polynomials is in $A_{0}\left[X_{1}, \ldots, X_{n+t}\right]$. If $A_{0} \subset A \subset K$ and $A$ has mixed characteristic, $\left(\prod_{i=1}^{d} B_{i}\right) H \in\left(F_{1}, \ldots, F_{d}\right)$ in $A\left[X_{1}, \ldots, X_{n+t}\right]$. Consequently, $\left(\Pi \bar{B}_{i}\right) \bar{H} \in\left(\bar{F}_{1}, \ldots, \bar{F}_{d}\right)$ in $S_{m}$, for every $m \in \max \operatorname{Spec}(A)$. Note that if $a \in A$ is the nonzero constant term of $\Pi B_{i}$, then $\overline{\Pi B}_{i} \in \mu S_{m}$ if and only if $m \in V(a)$ in $\operatorname{Spec} A$. Thus

$$
\bar{H} \in\left(\bar{F}_{1}, \ldots, \bar{F}_{d}\right)\left(S_{m}\right)_{\mu}
$$

whenever $m \in \operatorname{maxSpec}(A) \backslash V(a)$. The further assumption that $A$ be finitely generated over $Z$ guarantees that $\max \operatorname{Spec}(A) \backslash V(a)$ is both open and nonempty (hence, dense).

LEMMA 2.5. Let $I=\left(F_{1}, \ldots, F_{d}\right) \subset \mu S$ and let $Y_{1}, \ldots, Y_{S}$ be polynomials which form a regular sequence in $I S_{\mu}$. Then there exists $A_{0} \subset K$ finitely generated over $Z$ having the property:

For every finitely generated Z-algebra $A$ between $A_{0}$ and $K,\left(F_{1}, \ldots, F_{d}\right) \subset$ $A\left[X_{1}, \ldots, X_{n+t}\right]$ and there is a dense open set $U$ in $\operatorname{maxSpec}(A)$ such that, for every $m \in U,\left\{\bar{Y}_{1}, \ldots, \bar{Y}_{s}\right\}$ forms a regular sequence in $\left(\bar{F}_{1}, \ldots, \bar{F}_{d}\right)\left(S_{m}\right)_{\mu}$.

Proof. Construct $A_{00}$ by adjoining all of the coefficients of the $F_{i}$ 's to $Z$. Since $Y_{i} \in\left(F_{1}, \ldots, F_{d}\right) S$, we can use Lemma 2.4 to construct $A_{0 i}$, finitely generated over $Z$, such that $\bar{Y}_{i} \in\left(\bar{F}_{1}, \ldots, \bar{F}_{d}\right) S_{m}$ is always valid when $m$ is in some dense open subset of the maxSpec of any finitely generated $Z$-algebra $A$ such that $A_{0 i} \subset A \subset K$. Extend $Y_{1}, \ldots, Y_{s}$ to a system of parameters $\left\{Y_{1}, \ldots, Y_{s}, Y_{s+1}, \ldots, Y_{n+t}\right\}$ for $\mu$ in $S_{\mu}$. Then for each $1 \leqslant j \leqslant n+t$, there exists $e_{j}$ such that $X_{j}^{e_{j}} \in\left(Y_{1}, \ldots, Y_{n+t}\right) S_{\mu}$. By Lemma 2.4 , we can construct rings $B_{0 j}$, for each $1 \leqslant j \leqslant n+t$ such that $\bar{X}_{j}^{e_{j}} \in$ $\left(\bar{Y}_{1}, \ldots, \bar{Y}_{n+t}\right)\left(S_{m}\right)_{\mu}$ (with of course the usual generality that $B$ is any finitely generated $Z$-algebra such that $B_{0 j} \subset B \subset K$, and the usual restriction to a dense open set of $m$ 's in $\operatorname{maxSpec}(B))$. Let $A_{0}$ be the common refinement of the rings $A_{0 i}$ and $B_{0 i}$ (i.e. $A_{0}$ is obtained by adjoining to $Z$ all the finitely many elements of $K$ necessary to construct each of the $A_{0 i}$ 's and $B_{0 j}$ 's.) In particular, $A_{0} \subset K$ is finitely generated over $Z$ and over each $A_{0 i}$ and $B_{0 i}$ ). The same is true for any $A \subset K$ which is finitely generated over $A_{0}$. Therefore, given any such $A$, there exist dense open sets $U_{i}$, for $1 \leqslant i \leqslant s$, and $W_{j}$, for $1 \leqslant j \leqslant n+t$, in $\operatorname{maxSpec}(A) \operatorname{such}$ that $\bar{Y}_{i} \in$ $\left(\bar{F}_{1}, \ldots, \bar{F}_{d}\right)\left(S_{m}\right)_{\mu}$ whenever $m \in U_{i}$ and $X_{j}^{e_{j}} \in\left(\bar{Y}_{1}, \ldots, \bar{Y}_{n+t}\right)\left(S_{m}\right)_{\mu}$ whenever $m \in$ $W_{j}$. Thus, if $m \in U=\bigcap_{i=1}^{s} U_{i} \cap \bigcap_{j=1}^{n+t} W_{j}$, then $\left\{\bar{Y}_{1}, \ldots, \bar{Y}_{n+t}\right\}$ must be a system of 
parameters for $\mu$ in $\left(S_{m}\right)_{\mu}$ and, so, $\left\{\bar{Y}_{1}, \ldots, \bar{Y}_{s}\right\}$ must be a regular sequence in $\left(\bar{F}_{1}, \ldots, \bar{F}_{d}\right)\left(S_{m}\right)_{\mu}$. It remains only to observe that $U$ is a dense open set because it is the intersection of finitely many such sets.

From now on, we will abbreviate the statement that "There exists $A_{0}$ such that for every $A \subset K$ fintiely generated over $A_{0}$, there is a dense open set $U$ such that for every $m \in U, \ldots$ " with the suggestive phrase "for almost every $m, \ldots$ ".

Lemma 2.6. Let $\operatorname{deg}\left(\bar{X}_{i}\right)$ be defined to be $\operatorname{deg}\left(X_{i}\right)$. Then every homogeneous polynomial $F \in S$ is homogeneous in $S_{m}$, and either $\operatorname{deg}(\bar{F})=\operatorname{deg}(F)$ or $\bar{F}=0$. Moreover, each $\bar{G}_{i} \neq 0$ for almost every $m$, and therefore, $R_{m}$ is a graded ring with the "same" grading as $R$ (i.e. for every $F_{i} \in S$, either $\operatorname{deg}(F)=\operatorname{deg}(f)=\operatorname{deg}(\bar{f})$ or $\bar{f} \equiv 0$ in $R)$, for almost every $m$.

Proof. By setting $\operatorname{deg}\left(\bar{X}_{i}\right)=\operatorname{deg}\left(X_{i}\right)$, obviously each monomial term of $F$ will have the same degree as each monomial term of $\bar{F}$. Thus $\operatorname{deg}(F)=\operatorname{deg}(\bar{F})$ unless every monomial term of $\bar{F}$ vanishes. If $m \notin \bigcup V\left(a_{i}\right)$, where $\left\{a_{i}\right\}_{i=1}^{N}$ runs through all the coefficients of $F$, then none of the monomial terms of $\bar{F}$ vanish. But $m \notin \cup V\left(a_{i}\right)$ for almost every $m$. In particular, for each $1 \leqslant i \leqslant t$, and for almost every $m$, none of the monomial terms of $\bar{G}_{i}$ vanish. Consequently, $R_{m}=S_{m} /\left(\bar{G}_{1}, \ldots, \bar{G}_{t}\right)$ has the "same" grading as $R=S /\left(G_{1}, \ldots, G_{t}\right)$ for almost every $m$.

LEMmA 2.7. If $\left(Y_{1}, \ldots, Y_{s}\right) \subset J \subset \mu$ where $J=J\left(G_{1}, \ldots, G_{t}\right)$ is the Jacobian ideal for $R$, and if $\left\{Y_{1}, \ldots, Y_{s}\right\}$ forms a regular sequence in $S_{\mu}$; then, for almost every $m$, $\left(\bar{Y}_{1}, \ldots, \bar{Y}_{s}\right) \subset J\left(\bar{G}_{1}, \ldots, \bar{G}_{t}\right) \subset \mu$ in $S_{m}$ and $\left\{\bar{Y}_{1}, \ldots, \bar{Y}_{s}\right\}$ forms a regular sequence in $\left(S_{m}\right)_{\mu}$.

Proof. This is an immediate corollary of Lemma 2.5 (which says that being a regular sequence inside a given ideal is "preserved" in passing to characteristic $p$ ) once we observe that the respective Jacobian ideal $J\left(G_{1}, \ldots, G_{t}\right)$ and $J\left(\bar{G}_{1}, \ldots, \bar{G}_{t}\right)$ have the "same" finite set of generators. Since the ideal of $t \times t$ minors of the $t \times(n+t)$ matrix $D_{i}\left(G_{j}\right)$ is the "same" as the ideal of $t \times(n+t)$ matrix $D_{i}\left(\bar{G}_{j}\right)$, there is only one possible difficulty-that $\left(\bar{G}_{1}, \ldots, \bar{G}_{t}\right)$ might not be a complete intersection ideal of height $t$, in which case, $J\left(\bar{G}_{1}, \ldots, \bar{G}_{t}\right)$ is not the ideal of $t \times t$ minors of $D_{i}\left(\bar{G}_{j}\right)$. However, another application of 2.5 shows that if $\left(G_{1}, \ldots, G_{t}\right)$ is a regular sequence in $\mu S$, then $\left(\bar{G}_{1}, \ldots, \bar{G}_{t}\right)$ is a regular sequence in $\mu S_{\mu}$ for almost every $m$.

We are now in a position to prove the characteristic 0 version of the main theorem:

THEOREM 2.8. Let $R=S \% I$ be a special graded $K$-algebra where $S=$ $K\left[X_{1}, \ldots, X_{n+t}\right]$ and $K$ has characteristic 0 . If $I$ is a complete intersection of height $t$ with homogeneous generators $\left(G_{1}, \ldots, G_{t}\right)$ and if $Y_{1}, \ldots, Y_{n}$ are homogeneous polynomials such that $\left\{y_{1}, \ldots, y_{n}\right\}$ forms a system of parameters for $\mu$ in $R$ and $\left(Y_{1}, \ldots, Y_{s}\right) \subset J\left(G_{1}, \ldots, G_{t}\right)$, then:

(1) $R$ has $F$-pure type if $a(R)<-\sum_{i=s+1}^{n} \operatorname{deg}\left(Y_{i}\right)$.

(2) $R$ does not have F-pure type if $a(R)>0$. 
Proof. Since $\left\{y_{1}, \ldots, y_{n}\right\}$ forms a homogeneous system of parameters for $\mu R$, $\left\{Y_{1}, \ldots, Y_{n}, G_{1}, \ldots, G_{t}\right\}$ is a maximal regular sequence in $\mu S_{\mu}$. By Lemma 2.5, then, $\left\{\bar{Y}_{1}, \ldots, \bar{Y}_{n}, \bar{G}_{1}, \ldots, \bar{G}_{t}\right\}$ is a maximal regular sequence in $\mu\left(S_{m}\right)_{\mu}$ for almost every $m$. Hence $\left(\bar{G}_{1}, \ldots, \bar{G}_{t}\right)$ is a complete intersection ideal of height $t$ in $S_{m}$ and $\left\{\bar{y}_{1}, \ldots, \bar{y}_{n}\right\}$ is a system of parameters for $\mu\left(R_{m}\right)_{\mu}$, for almost every $m$. Since the grading for both $S_{m}$ and $R_{m}$ is the "same" as for $S$ and $R$ (Lemma 2.5),

$$
a(R)=\sum_{i=1}^{t} \operatorname{deg}\left(G_{i}\right)-\sum_{i=1}^{n+t} \operatorname{deg}\left(Y_{i}\right)=\sum_{i=1}^{t} \operatorname{deg}\left(\bar{G}_{i}\right)-\sum_{i=1}^{n+t} \operatorname{deg}\left(\bar{Y}_{i}\right)=a\left(R_{m}\right),
$$

for almost every $m$. In particular, $a(R)<-\sum_{i=s+1}^{n} \operatorname{deg}\left(Y_{i}\right)$ implies $a\left(R_{m}\right)<$ $-\sum_{i=s+1}^{n} \operatorname{deg}\left(\bar{Y}_{i}\right)$, for almost every $m$. Finally the fact that $\left(Y_{1}, \ldots, Y_{s}\right)$ lies in $J\left(G_{1}, \ldots, G_{t}\right)$ and forms a homogeneous regular sequence in $S_{\mu}$ guarantees that $\left(\bar{Y}_{1}, \ldots, \bar{Y}_{s}\right) \subset J\left(\bar{G}_{1}, \ldots, \bar{G}_{t}\right)$ and forms a homogeneous regular sequence in $\left(S_{m}\right)_{\mu}$ (Lemma 2.7) for almost every $m$. Taking advantage, as usual, of the fact that the finite "intersection" of "for almost every $m$ " holds for almost every $m$, we see that all the hypotheses of Theorem 2.1 are satisfied for almost every $m$. Hence, either $R_{m}$ is $F$-pure or $R_{m}$ has characteristic $p<\sum_{i=1}^{s} \operatorname{deg}\left(\bar{Y}_{i}\right)=\sum_{i=1}^{s} \operatorname{deg}\left(Y_{i}\right)$, for almost every $m$. But, of course, the characteristic of $R_{m}$ is greater than $\sum_{i=1}^{s} \operatorname{deg}\left(Y_{i}\right)$ whenever $m \in \max \operatorname{Spec}(A) \backslash V\left(\prod_{i=1}^{N} Q_{i}\right)$ where $\left\{Q_{i}\right\}_{i=1}^{N}$ is the list of all prime numbers less than or equal to $\sum_{i=1}^{s} \operatorname{deg}\left(Y_{i}\right)$. Thus $R_{m}$ must be $F$-pure for almost every $m$. That is, $R$ has $F$-pure type.

The partial converse that $R$ does not have $F$-pure type if $a(R)>0$ was proved by Hochster and Roberts [5]. It follows from the fact that $a(R)=a\left(R_{m}\right)$ for almost every $m$ and from Remark 2.2 .

REMARK 2.9. In the case where the irrelevant maximal ideal of $R$ is actually generated by forms of degree 1 (the truly homogeneous case) the condition $a(R)<$ $-\sum_{i=s+1}^{n} \operatorname{deg}\left(Y_{i}\right)$ becomes $a(R)<s-n$ the negative "cograde" of the ideal $J$.

Corollary 2.10. Let $R$ be as in the hypothesis of Theorem 2.8. If, in addition, $R$ has an isolated singularity at $\mu$, then:

(1) $R$ is $F$-pure type if $a(R)<0$.

(2) $R$ is not F-pure type if $a(R)>0$.

Proof. The singularity at $\mu$ is isolated if and only if the Jacobian ideal of $R$ contains a maximal homogeneous system of parameters, $\left\{Y_{1}, \ldots, Y_{n}\right\}$. In this case $s=n$ and $\sum_{i=s+1}^{n} \operatorname{deg}\left(Y_{i}\right)=0$.

I would like to thank the referee whose reading of the text was careful and helpful despite several confusing typographical errors.

\section{REFERENCES}

1. J. A. Eagon and M. Hochster, R-sequences and indeterminates, Quart. J. Math Oxford Ser. 25 (1974), $61-71$.

2. R. Fedder, F-purity and rational singularity, Trans. Amer. Math. Soc. 278 (1983), 461-480.

3. S. Goto and K.-i. Watanabe, The structure of 1-dimensional F-pure rings, J. Algebra 49 (1977), 415-421.

4. M. Hochster and J. L. Roberts, Rings of invariants of reductive groups acting on regular rings are Cohen-Macaulay, Adv. in Math. 13 (1974), 115-175. 
5.

6. M. Hochster, Cyclic purity versus purity in excellent Noetherian rings, Trans. Amer. Math. Soc. 231 (1977), 463-488.

7. E. Matlis, Injective modules over Noetherian rings, Pacific J. Math. 8 (1958), 511-528. MR 20 \# 5800 .

8. K.-i. Watanabe, Rational singularities with $K^{*}$-action, Proc. Trente Conf., Lecture Notes in Pure and Appl. Math. 84, Dekker, New York; 1983

Department of Mathematics, University of Missouri, Columbia, Missouri 65211 